\title{
NEPHROTOXICITY IN ADULT PATIENTS UNDERGOING HYPERTHERMIC INTRAPERITONEAL CHEMOTHERAPY AND CYTOREDUCTIVE SURGERY
}

\author{
Etulain Santos MJ, Canal MI, Gago S, Cabrerizo P, Jiménez C, Zaballos M. \\ General University Hospital Gregorio Marañón. Madrid, Spain
}

\section{Background and Goal of Study}

Cytoreductive surgery associated with hyperthermic intraperitoneal chemotherapy (CRS + HIPEC) may alter postoperative renal function, causing an impact in morbidity, mortality and cost of this procedure. Our aim was to examine the association of postoperative changes in renal function according to RIFLE criteria in patients who underwent CRS+HIPEC. RIFLE is a new definition of acute kidney injury classification based on changes in serum creatinine levels or the duration and severity of decline in the urine

\section{Materials and methods}

After committee approval, a retrospective cohort study was performed evaluating patients undergoing HIPEC from 2011 to 2015. Postoperative renal function was assessed using serial serum creatinine measurements and was based on serum creatinine changes from pre-operative values, according to the risk, injury, failure, loss, end-stage kidney (RIFLE) classification.

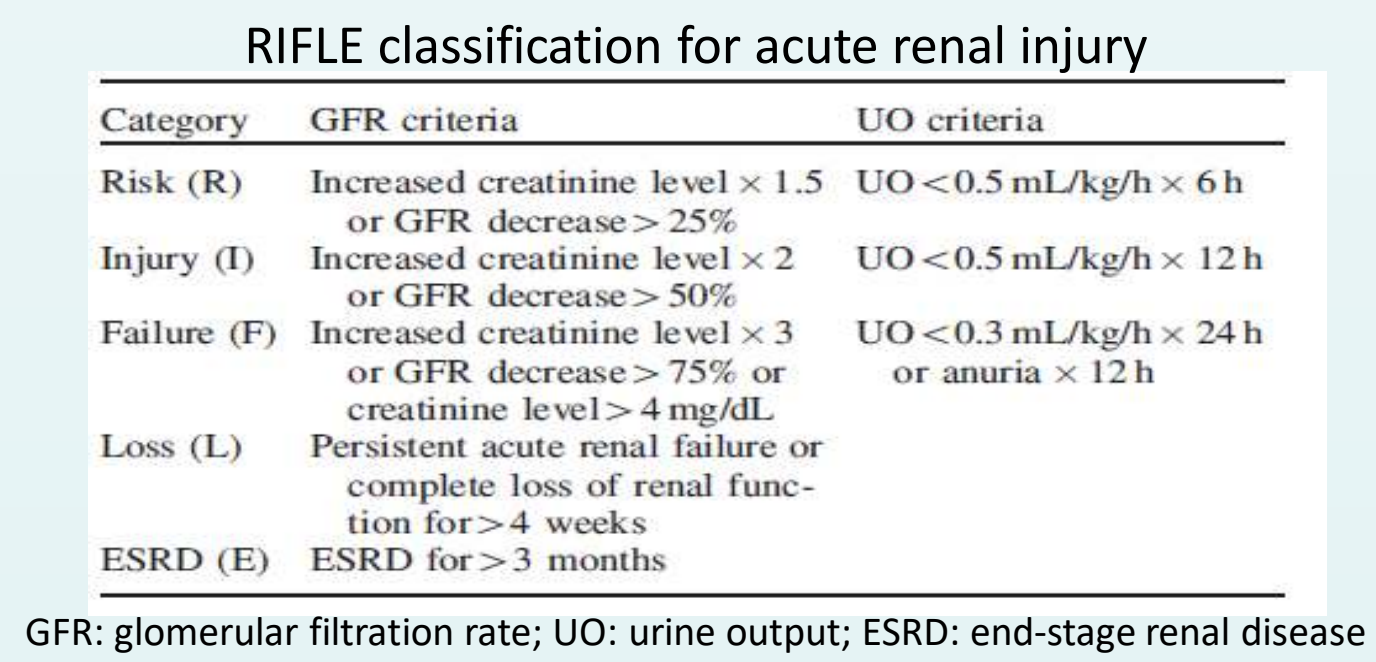

\section{Results and discussion}

We evaluated 257 patients, ASA I-III with a mean age of $56 \pm 11$ years. The median and range of the peritoneal carcinomatosis index was 13(6-23). HIPEC time was $30 \mathrm{~min}$ in $44 \%$ of patients, $60 \mathrm{~min}$ in $35 \%$ of cases and in a $21 \%$ of patients HIPEC time lasted 90 min. Postoperative acute changes in renal function were observed in 29 patients (11.1\%); (Risk: $n=10$; Injury: $n=9$; Failure: $n=8$; Loss: $n=2$ ). Relative to the antineoplastic agent, no changes in renal function were observed in patients who received mitomicyn $C(n=62) ; 7$ of 101 patients who received oxaliplatin presented renal changes; 21 of $92(22.8 \%)$ of patients who received cisplatin presented renal dysfunction $(p<0.0001)$. We did not observe differences in renal dysfunction in the years evaluated.

\begin{tabular}{|c|c|c|c|c|c|}
\hline RENAL FAILURE & MITOMICIN n=62 & OXALIPLATIN n=101 & CISPLATIN n=92 & \multicolumn{2}{|c|}{257 PATIENTS } \\
\hline No changes (\%) & 100 & 93 & 77,2 & \multicolumn{2}{|c|}{$228(88,72 \%)$} \\
\hline Risk (\%) & 0 & 4 & 5,4 & $\mathbf{R}$ & $10(3,89 \%)$ \\
\hline Injury (\%) & 0 & 0 & 9,8 & 1 & $(3,5 \%)$ \\
\hline Failure (\%) & 0 & 3 & 5,4 & $\mathbf{F}$ & $8(3,11 \%)$ \\
\hline Loss (\%) & 0 & 0 & 2,2 & $\mathbf{L}$ & $2(0,07 \%)$ \\
\hline \multirow[t]{2}{*}{ ESRD (\%) } & 0 & 0 & 0 & $E$ & $\%$ \\
\hline & $p=$ & 0,0001 & & & \\
\hline
\end{tabular}

\begin{tabular}{|c|c|c|}
\hline & Porcentage, mean or median & $N=257$ \\
\hline Age & $56 \pm 11$ (media+DS) & \\
\hline Gender (M/F) & $32 / 68$ & $83 / 174$ \\
\hline \multicolumn{3}{|l|}{ ASA } \\
\hline I & 9 & 22 \\
\hline II & 65 & 163 \\
\hline III & 26 & 64 \\
\hline Comorbidity & 67 & 172 \\
\hline \multicolumn{3}{|l|}{ PS } \\
\hline 1 & 65 & 164 \\
\hline 2 & 34 & 87 \\
\hline 3 & 1 & 2 \\
\hline \multicolumn{3}{|l|}{ Primary Tumor } \\
\hline Appendix & 26 & 66 \\
\hline Colorectum & 34 & 86 \\
\hline Gynecol & 27 & 68 \\
\hline Gastric & 4,4 & 11 \\
\hline Mesothelioma & 3,6 & 9 \\
\hline Sarcoma & 3,6 & 9 \\
\hline Preop Creatinin & $0.74 \pm 0.19$ & $($ mean $+S D)$ \\
\hline Preop Urea & $34 \pm 9.15$ & $($ mean $+\mathrm{SD})$ \\
\hline PCl & $13(6-23)$ & th percentile) \\
\hline IO blood transfusions & $0(0-1)$ & th percentile) \\
\hline CC score & $\%$ & $\mathrm{~N}$ \\
\hline 0 & 75 & 184 \\
\hline 1 & 22 & 54 \\
\hline 2 & 1,2 & 3 \\
\hline 3 & 1,6 & 4 \\
\hline DRUG HIPEC & $\%$ & N \\
\hline Mitomicin & 24 & 62 \\
\hline Oxaliplatin & 39 & 101 \\
\hline Cisplatin & 36 & 92 \\
\hline Paclitaxel & 1 & 2 \\
\hline Tiempo HIPEC & $\%$ & N \\
\hline $30 \mathrm{~min}$ & 44 & 111 \\
\hline $60 \mathrm{~min}$ & 35 & 88 \\
\hline $90 \mathrm{~min}$ & 21 & 56 \\
\hline Temp HIPEC & $\%$ & N \\
\hline$<42 \circ \mathrm{C}$ & 4 & 10 \\
\hline$>42 \circ \mathrm{C}$ & 96 & 246 \\
\hline IO Fluids & mean $\pm S D$ & \\
\hline${ }^{*}$ Total volume $10 \mathrm{~mL}$ & $5970 \pm 1615$ & \\
\hline *Cristaloids $\mathrm{mL}$ & $5205 \pm 1481$ & \\
\hline *Coloids mL & $785 \pm 407$ & \\
\hline Orine output MI & mean $\pm S D$ & \\
\hline *Total mL & $1302 \pm 568$ & \\
\hline *HIPEC mL & $732 \pm 324$ & \\
\hline
\end{tabular}

Conclusions: Postoperative nephrotoxicity can complicate CRS/HIPEC. Permanent renal dysfunction was not a common complication $(0.8 \%)$ in our cohort. Cisplatin was the principal chemotherapy agent associated with renal dysfunction in patients undergoing CRS/HIPEC 